\title{
How small can ground movements be to be detectable with GNSS?
}

\section{Other Conference Item}

\section{Author(s):}

Hohensinn, Roland; Ruttner, Pia; Soja, Benedikt (D); Rothacher, Markus

Publication date:

2021-04

Permanent link:

https://doi.org/10.3929/ethz-b-000528785

\section{Rights / license:}

Creative Commons Attribution 4.0 International

\section{Originally published in:}

EGUsphere, https://doi.org/10.5194/egusphere-egu21-1999 
EGU21-1999

https://doi.org/10.5194/egusphere-egu21-1999

EGU General Assembly 2021

(C) Author(s) 2022. This work is distributed under

the Creative Commons Attribution 4.0 License.

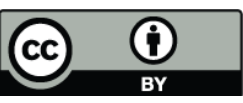

\section{How small can ground movements be to be detectable with GNSS?}

Roland Hohensinn, Pia Ruttner, Benedikt Soja, and Markus Rothacher

ETH Zurich, Institute of Geodesy and Photogrammetry, Zürich, Switzerland (rolandh@ethz.ch)

High-precision GNSS (Global Navigation Satellite System) positioning can reach an accuracy at the millimeter level, and by collecting these data over years, very small ground movements can be resolved. These data can be used to study geodynamics, like continental drifts, tidal- and non-tidal loading effects, or earthquakes, for example. Here we focus on the sensitivity of GNSS for resolving these different types of movements. We derive minimal detectable displacements for linear drift rates, annual- and semiannual periodic motions, and offsets - these are main parameters of the so-called standard linear trajectory model for GNSS station motions. For our analysis, our data comes from several hundreds of permanent GNSS stations across Europe- the GNSS stations' coordinates are obtained at a daily sampling rate, with almost 25 years of data available for some stations. Based on cleaned residual GNSS time series we calibrate a "power-law plus white noise" stochastic model for each station. Together with the functional trajectory model we compute the formal errors of the movement parameters based on a least-squares adjustment. Based on these errors we then introduce the statistics to derive minimum detectable displacements for the movement parameters.

Our analysis shows that the minimum detectable trends can be as low as few tenth of millimeters per year. The minimum detectable amplitudes at the annual and semiannual periods are at the millimeter level or lower, and the detectable offset is few millimeters on average, too. As expected, the minimum detectable displacements depend strongly on the length of the datasets and on the noise characteristics. Another important parameter is the number of discontinuities and offsets for each station - it impacts the minimum detectable trend. We conclude that such an analysis can be very useful for sensitivity studies in climate change monitoring. Furthermore, the methodology cannot only be applied in the field of GNSS time series analysis, but also to any other time series data in geosciences. 